\title{
Evasive maneuvers for a sequential fourth generation to circumvent the Higgs data
}

\section{Dipankar Das*t}

Indian Institute of Technology (Indore), Khandwa Road, Simrol, Indore 453552, India

E-mail: d.daseiti.ac.in

\begin{abstract}
So far, we have been unable to determine the sign of the down-type Yukawa couplings from the current Higgs data. We use this freedom to accommodate extra generations of fermions that obtain their masses through the exact same mechanism as the first three generations. Our recipe successfully hides the extra fermionic generations not only in the Higgs boson production through gluon fusion, but also in the loop induced Higgs decays like $h \rightarrow \gamma \gamma$ and $h \rightarrow Z \gamma$. We also illustrate how such hidden extra generation of fermions may unravel themselves in the di-Higgs production mechanisms.
\end{abstract}

Corfu Summer Institute 2019 "School and Workshops on Elementary Particle Physics and Gravity" (CORFU2019)

31 August - 25 September 2019

Corfu, Greece

\footnotetext{
* Speaker.

${ }^{\dagger}$ Work done in collaboration with Anirban Kundu and Ipsita Saha.
} 


\section{Why evasive maneuver?}

After the first announcement of the discovery of a Higgs-like particle in July, 2012 at the LHC [1, 2], the data has evolved substantially to vindicate the Standard Model (SM) with an impressive accuracy [3]. The level of agreement of the Higgs data with the corresponding SM prediction has led the community to converge to the view that the observed resonance must be an SM-like Higgs scalar, if not the SM Higgs scalar itself. The fact that the LHC Higgs data is gradually drifting towards the SM expectations has pushed many scenarios that go beyond the SM (BSM) to some contrived corner of the parameter space. For certain BSMs, the situation is even worse as they have nowhere to hide, because quantum effects coming from some of the heavy degrees of freedom do not decouple and hence they leave observable imprints in the Higgs signal strengths. The SM extended by a chiral fourth generation (SM4) [4] constitutes such an example. The fourth generation quark masses, so heavy as to avoid the direct detection bound, are proportional to the corresponding Yukawa couplings and thus, their contributions to the $g g \rightarrow h$ production amplitude saturate to a constant value just as in the case of the top quark loop. To illustrate this point more

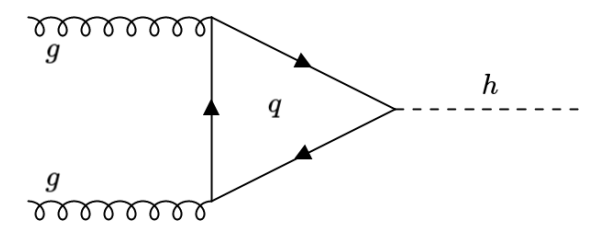

Figure 1: Higgs production via gluon fusion.

explicitly, we first define the Higgs coupling modifiers as follows:

$$
\kappa_{X}=\frac{g_{X X h}}{\left(g_{X X h}\right)^{\mathrm{SM}}},
$$

where, $X$ is a generic notation for a massive vector boson or a charged fermion. With this, we can write down the modification factor for $g g \rightarrow h$ production cross section, in the case of SM4, as follows:

$$
\mathscr{R}_{g}=\frac{\left|\sum_{f=t, t^{\prime}, b^{\prime}} \kappa_{f} \mathscr{A}\left(\tau_{f}\right)\right|^{2}}{\left|\mathscr{A}\left(\tau_{t}\right)\right|^{2}},
$$

where the extra contributions from the fourth generation of quarks in the Feynman diagram of Fig. 1 have been taken into account. Using $\tau_{f}=\left(2 m_{f} / m_{h}\right)^{2}$ the loop function is given by[5]

$$
\mathscr{A}\left(\tau_{f}\right)=-2 \tau_{f}\left[1+\left(1-\tau_{f}\right) f\left(\tau_{f}\right)\right]
$$

where, for heavy fermions $\left(\tau_{f}>1\right)$, we have

$$
f\left(\tau_{f}\right)=\left[\sin ^{-1}\left(\sqrt{1 / \tau_{f}}\right)\right]^{2}
$$

The interesting fact to note is that, in the limit $m_{f} \gg m_{h}$, i.e., $\tau_{f} \rightarrow \infty$, the loop function $\mathscr{A}\left(\tau_{f}\right)$ saturates to a constant value of $-4 / 3$. Therefore, in the heavy mass limit, the loop function can be factored out in the numerator of Eq. (1.2), leaving behind,

$$
\mathscr{R}_{g} \approx\left|\kappa_{t}+\kappa_{t^{\prime}}+\kappa_{b^{\prime}}\right|^{2} \text {. }
$$


Thus, in the case of SM4 where $t, t^{\prime}$ and $b^{\prime}$ are treated identically in their Yukawa couplings, we have $\kappa_{t}=\kappa_{t^{\prime}}=\kappa_{b^{\prime}}=1$ which implies $\mathscr{R}_{g} \approx 9$. Such an enhancement in the Higgs production would have been difficult to miss at the LHC. Since no significant deviation of the Higgs production crosssection from the SM expectations has been observed, we are led to believe that the possibility of a sequential fourth generation is strongly disfavored from the existing data $[6,7,8,9,10]$.

There have been efforts to moderate the enhanced production in the ggF channel by reducing the individual branching ratios (BRs) of the Higgs boson into different visible channels. This can be achieved, e.g., by adjusting the mass of the fourth generation neutrino $\left(m_{v^{\prime}}\right)$ so that the Higgs boson mainly decays invisibly into a pair of fourth generation neutrinos thereby increasing the total decay width of the Higgs boson [11, 12, 13]. However, we take on a more ambitious task of accommodating extra generations of fermions which couple to the SM Higgs in an identical manner as the first three generations, without altering appreciably the Higgs signal strengths from their corresponding SM expectations. As will be explained, this is feasible because the Yukawa couplings of up and down-type fermions can be so arranged as to make the extra contributions cancel out. Such a cancellation, quite remarkably, is also effective for loop-induced Higgs decays. Such a conspiracy of the couplings will be allowed thanks to our inability to pin down the downtype Yukawa couplings from the current Higgs data. However, a practical realization of this will not be possible unless we extend the scalar sector of the SM.

\section{The maneuver}

To accommodate extra sequential generations of fermions without substantially altering the Higgs signal strengths from their corresponding SM expectations, we will take advantage of the fact that the sign of the down-type quark Yukawas as well as that of the charged lepton Yukawas are yet to be probed experimentally. To this end, we note from Eq. (1.2) that the new physics (NP) contribution to $g g \rightarrow h$ production cross section is proportional to $\left(\kappa_{t^{\prime}}+\kappa_{b^{\prime}}\right)$. Quite clearly, the NP contribution gets canceled in the heavy mass limit if $\kappa_{t^{\prime}}=-\kappa_{b^{\prime}}$. With this in mind, we define the wrong sign limit as follows:

$$
\begin{aligned}
\kappa_{V}=1 & (V=W, Z) \\
\kappa_{u}=1 & \text { (for up type quarks) } \\
\kappa_{d}=-1 & \text { (for down type quarks) } \\
\kappa_{\ell}=-1 & \text { (for charged leptons). }
\end{aligned}
$$

Recall that, the relative sign between $\kappa_{V}$ and $\kappa_{u}$ can be fixed from the interference of the $W$-loop and the top-loop in the $h \rightarrow \gamma \gamma$ decay amplitude. Clearly, in the wrong-sign limit, the NP contribution to the $g g \rightarrow h$ production amplitude will disappear for heavy masses of the fermions making the production cross-section consistent with the SM prediction. Now, the new fermions will also affect the loop induced Higgs decays like $h \rightarrow \gamma \gamma$ and at this point, it is not very obvious that the wrongsign trick will also help moderate the deviation of the Higgs to diphoton signal strength from the corresponding SM prediction. But, one should remember that, while computing the $h \rightarrow \gamma \gamma$ decay width, in addition to the quarks, the contributions from the fourth generation charged lepton $\left(\tau^{\prime}\right)$ also needs to be taken into account. Consequently, the NP contribution to the $h \rightarrow \gamma \gamma$ amplitude, in 
the heavy mass limit, is proportional to

$$
\kappa_{\gamma \gamma}=\sum_{f=t^{\prime}, b^{\prime}, \tau^{\prime}} Q_{f}^{2} N_{c}^{f} \kappa_{f}
$$

where, $Q_{f}$ is the electric charge of the fermion $f$, and the color factor $N_{c}^{f}=3$ for quarks and 1 for leptons. Similarly, for the decay $h \rightarrow Z \gamma$, the NP contribution from the fourth generation of fermions, will be proportional to

$$
\kappa_{Z \gamma}=\sum_{f=t^{\prime}, b^{\prime}, \tau^{\prime}} Q_{f} T_{3}^{f} N_{c}^{f} \kappa_{f}
$$

where $T_{3}^{f}$ denotes the isospin projection of $f_{L}$. One can easily check that in the wrong sign limit defined by Eqs. (2.1)-(2.4), $\kappa_{\gamma \gamma}=\kappa_{Z \gamma}=0$. Therefore, the extra generations of fermions can remain completely hidden from the Higgs data in the wrong sign limit.

\section{Implementation}

Now that we have established how extra sequential generations of fermions can escape detection in the single Higgs phenomenology, it's time to build a theoretical framework where the conspiracy of couplings given in Eqs. (2.1)-(2.4) can be achieved. The SM relies on the minimalistic scenario with only one scalar doublet. In this case, the phases of the Yukawa couplings in Eqs. (2.3) and (2.4) can be rotated away using redefinitions of fields. Moreover, the relative sign between the $W W h$ coupling and $f f h$ Yukawa coupling can fixed in the SM from tree-unitarity [14].

Therefore, we need to extend the scalar sector of the SM to implement the wrong sign limit. As it happens, we do not need to look far - a type II two Higgs-doublet model (2HDM) can easily accommodate a wrong sign limit. In this variant of $2 \mathrm{HDM}$, one of the doublets $\left(\phi_{2}\right)$ couples only to the fermions with $T_{3}=+1 / 2$ while the other doublet $\left(\phi_{1}\right)$ couples only to the $T_{3}=-1 / 2$ fermions. this arrangement ensures the absence of scalar mediated flavor changing neutral currents at the tree-level. Note that we are implicitly introducing right-handed neutrinos and allowing Dirac mass terms only. In this set-up, the coupling modifiers for the SM-like Higgs scalar $(h)$ are given by

$$
\begin{aligned}
\kappa_{V}=\sin (\beta-\alpha), \quad(V=W, Z) & \\
\kappa_{u}=\sin (\beta-\alpha)+\cot \beta \cos (\beta-\alpha), & \text { (for up type quarks) } \\
\kappa_{d}=\sin (\beta-\alpha)-\tan \beta \cos (\beta-\alpha), & \text { (for down type quarks) } \\
\kappa_{\ell}=\sin (\beta-\alpha)-\tan \beta \cos (\beta-\alpha), & \text { (for charged leptons) }
\end{aligned}
$$

where, following the usual convention $[15,16], \tan \beta=v_{2} / v_{1}$ is the ratio of the two vacuum expectation values (VEVs) and $\alpha$ is the mixing angle in the CP-even scalar sector. Note that, the condition $[17,18,19,20,21]$

$$
\cos (\beta-\alpha)=\frac{2}{\tan \beta}, \quad \text { with, } \tan \beta \gg 2
$$

will lead us to the desired wrong sign limit of Eqs. (2.1)-(2.4). 


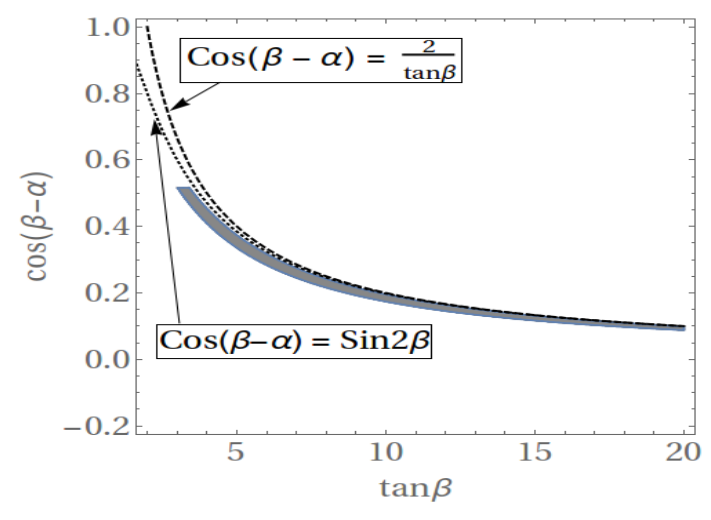

Figure 2: The gray shaded region, based on the benchmark given in Eq. (3.6), is allowed at $95 \%$ C.L. from data for Higgs signal strengths [22], and $\Delta S, \Delta T$ for the benchmark of Eq. (3.6). The dashed and dotted lines represent the contours for $\cos (\beta-\alpha)=2 / \tan \beta$ and $\cos (\beta-\alpha)=\sin 2 \beta$ respectively. The figure has been taken from Ref. [23].

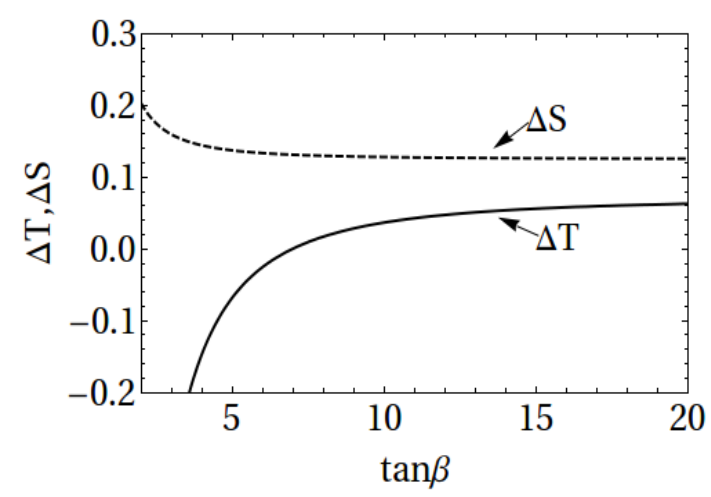

Figure 3: Variation of the NP contributions to the $S$ and $T$ parameters with $\tan \beta$ assuming the validity of Eq. (3.5). This plot has been obtained for the benchmark values of Eq. (3.6). The figure has been taken from Ref. [23].

Now, let us try to see explicitly whether our proposition passes through the constraints from the Higgs data. For this, we choose the following benchmark values for the nonstandard masses:

$$
\begin{aligned}
& m_{t^{\prime}}=550 \mathrm{GeV}, m_{b^{\prime}}=510 \mathrm{GeV}, m_{\tau^{\prime}}=400 \mathrm{GeV}, m_{v^{\prime}}=200 \mathrm{GeV}, \\
& m_{H}=400 \mathrm{GeV}, m_{A}=810 \mathrm{GeV}, m_{H+}=600 \mathrm{GeV}
\end{aligned}
$$

Note that, for such heavy masses, the signal strengths of the SM-like Higgs scalar will depend only on two parameters, $\beta$ and $\alpha$. Thus, in Fig. 2 the shaded area represents the 95\% C.L. allowed region from the Higgs signal strength measurements [22]. Since this figure is essentially independent of the precise values for the nonstandard masses, we could have conveniently chosen them to be heavy enough to evade the relevant bounds from direct searches [24]. From Fig. 2 we can easily see that the allowed region closely follows the contour of Eq. (3.5) which defines the wrong-sign limit in a type II 2HDM.

Moreover, we should also recall that, before the Higgs discovery, stringent constraints were placed on the sequential fourth generation of fermions $[4,25]$ from the electroweak precision parameters. But, in these studies, contributions from the fourth generation of fermions only were taken into consideration. However, in our case, the effects of the nonstandard scalars need to be taken into account too $[26,27,28]$. Consequently, the tight constraints brought in by the fourth generation of fermions can be easily diluted by suitable choice of the nonstandard scalar masses. We have explicitly displayed this feature in Fig. 3.

\section{Unraveling the extra generations}

So far, we have demonstrated that in the wrong-sign limit sequential extra generations of fermions can remain hidden from the single-Higgs phenomenology. Therefore, it is reasonable to 


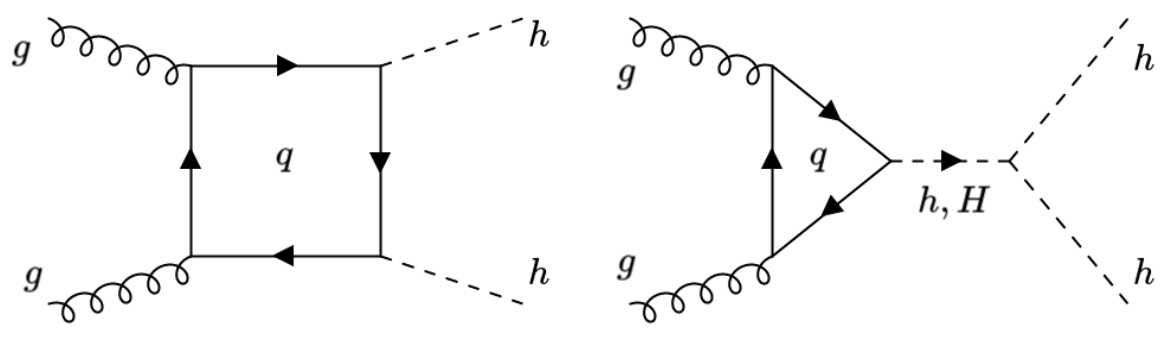

Figure 4: Double Higgs production via gluon fusion.

investigate how such hidden generations of fermions can possibly be probed in the experiments. As it happens, such a hidden fourth generation can be unveiled via the double Higgs production mechanism through gluon fusion, $g g \rightarrow h h$. The relevant Feynman diagrams have been displayed in Fig. 4. The first diagram depicts an amplitude that proceeds through a quark box-loop while the second diagram involves a quark triangle-loop and subsequent triple-Higgs interaction. In the SM, the main contribution comes from the top-quark loop. In our model, additional contributions arising from the $t^{\prime}$ and $b^{\prime}$ quarks need to be included also. Looking at the Feynman diagrams of Fig. 4 , we can easily see that the $t^{\prime}$ and $b^{\prime}$ contributions will add up in the box diagram because the Yukawa vertex appears twice in that diagram. Furthermore, in the heavy-mass limit, the $t^{\prime}$ and $b^{\prime}$ contributions will saturate and will roughly be equal to the contribution from the top-quark. Clearly, the box amplitude will pick up a factor of 3 compared to that in the SM. Since the box diagram dominates the $g g \rightarrow h h$ amplitude, the production cross-section will be enhanced roughly by a factor of 9. Such a huge enhancement implies that either a positive signal of di-Higgs production is seen in the early next run of the LHC, or the model is ruled out [29].

\section{Summary}

In summary, using the fact that the signs of the down-type Yukawa couplings have not been experimentally determined yet, we proposed a general recipe to hide one, or more, sequential generations of fermions from the single-Higgs phenomenology. At the same time, we have also explained how the presence of such hidden fermionic generations may be revealed in the di-Higgs production mechanism. In this context, it is worth emphasizing that probing the trilinear selfcoupling of the Higgs bosons has, so far, been the driving motivation behind the search for diHiggs production at the LHC. Quite evidently, we can add to the stimulus for di-Higgs searches by making this channel a sensitive tool for unveiling certain new physics models. In this short report, we have done precisely that. It has been shown that, an extra sequential generation of fermions with wrong-sign Yukawa couplings, which can remain completely hidden in single Higgs production and decays, can potentially reveal themselves exclusively in the di-Higgs searches. We hope that this will encourage our experimental colleagues to view the di-Higgs searches in a new light.

\section{References}

[1] CMS Collaboration, S. Chatrchyan et al., Observation of a new boson at a mass of $125 \mathrm{GeV}$ with the 
CMS experiment at the LHC, Phys. Lett. B716 (2012) 30-61, [arXiv: 1207. 7235].

[2] ATLAS Collaboration, G. Aad et al., Observation of a new particle in the search for the Standard Model Higgs boson with the ATLAS detector at the LHC, Phys. Lett. B716 (2012) 1-29, [arXiv:1207.7214].

[3] CMS Collaboration, A. M. Sirunyan et al., Combined measurements of Higgs boson couplings in proton-proton collisions at $\sqrt{s}=13 \mathrm{TeV}$, Eur. Phys. J. C79 (2019), no. 5 421, [arXiv:1809.10733].

[4] G. D. Kribs, T. Plehn, M. Spannowsky, and T. M. P. Tait, Four generations and Higgs physics, Phys. Rev. D76 (2007) 075016, [arXiv: 0706 .3718].

[5] J. F. Gunion, H. E. Haber, G. L. Kane, and S. Dawson, The Higgs Hunter's Guide, Front. Phys. 80 (2000) 1-404.

[6] O. Eberhardt, G. Herbert, H. Lacker, A. Lenz, A. Menzel, U. Nierste, and M. Wiebusch, Impact of a Higgs boson at a mass of $126 \mathrm{GeV}$ on the standard model with three and four fermion generations, Phys. Rev. Lett. 109 (2012) 241802, [arXiv:1209.1101].

[7] A. Djouadi and A. Lenz, Sealing the fate of a fourth generation of fermions, Phys. Lett. B715 (2012) 310-314, [arXiv:1204.1252].

[8] M. I. Vysotsky, The rise and fall of the fourth quark-lepton generation, in Proceedings, Helmholtz International Summer School on Physics of Heavy Quarks and Hadrons (HQ 2013): JINR, Dubna, Russia, July 15-28, 2013, pp. 311-316, 2014. arXiv:1312.0474.

[9] E. Kuflik, Y. Nir, and T. Volansky, Implications of Higgs searches on the four generation standard model, Phys. Rev. Lett. 110 (2013), no. 9 091801, [arXiv:1204.1975].

[10] A. Lenz, Constraints on a fourth generation of fermions from Higgs Boson searches, Adv. High Energy Phys. 2013 (2013) 910275.

[11] W.-Y. Keung and P. Schwaller, Long Lived Fourth Generation and the Higgs, JHEP 06 (2011) 054, [arXiv:1103.3765].

[12] A. Aparici, J. Herrero-Garcia, N. Rius, and A. Santamaria, On the Nature of the Fourth Generation Neutrino and its Implications, JHEP 07 (2012) 030, [arXiv: 1204 . 1021].

[13] M. Geller, S. Bar-Shalom, G. Eilam, and A. Soni, The $125 \mathrm{GeV}$ Higgs in the context of four generations with 2 Higgs doublets, Phys. Rev. D86 (2012) 115008, [arXiv:1209.4081].

[14] G. Bhattacharyya, D. Das, and P. B. Pal, Modified Higgs couplings and unitarity violation, Phys. Rev. D87 (2013) 011702, [arXiv: 1212.4651].

[15] G. C. Branco, P. M. Ferreira, L. Lavoura, M. N. Rebelo, M. Sher, and J. P. Silva, Theory and phenomenology of two-Higgs-doublet models, Phys. Rept. 516 (2012) 1-102, [arXiv:1106.0034].

[16] G. Bhattacharyya and D. Das, Scalar sector of two-Higgs-doublet models: A minireview, Pramana 87 (2016), no. 3 40, [arXiv:1507.06424].

[17] P. M. Ferreira, R. Guedes, J. F. Gunion, H. E. Haber, M. O. P. Sampaio, and R. Santos, The Wrong Sign limit in the 2HDM, in Proceedings, 2nd Conference on Large Hadron Collider Physics Conference (LHCP 2014): New York, USA, June 2-7, 2014, 2014. arXiv:1410.1926.

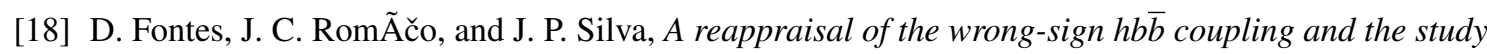
of $h \rightarrow Z \gamma$, Phys. Rev. D90 (2014), no. 1 015021, [arXiv: 1406.6080 ]. 
[19] P. M. Ferreira, R. Guedes, M. O. P. Sampaio, and R. Santos, Wrong sign and symmetric limits and non-decoupling in 2HDMs, JHEP 12 (2014) 067, [arXiv: 1409.6723 ].

[20] A. Biswas and A. Lahiri, Alignment, reverse alignment, and wrong sign Yukawa couplings in two Higgs doublet models, Phys. Rev. D93 (2016), no. 11 115017, [arXiv: 1511 . 07159].

[21] P. M. Ferreira, J. F. Gunion, H. E. Haber, and R. Santos, Probing wrong-sign Yukawa couplings at the LHC and a future linear collider, Phys. Rev. D89 (2014), no. 11 115003, [arXiv: 1403.4736 ].

[22] ATLAS, CMS Collaboration, G. Aad et al., Measurements of the Higgs boson production and decay rates and constraints on its couplings from a combined ATLAS and CMS analysis of the LHC pp collision data at $\sqrt{s}=7$ and $8 \mathrm{TeV}$, JHEP 08 (2016) 045, [arXiv: 1606.02266 ].

[23] D. Das, A. Kundu, and I. Saha, Higgs data does not rule out a sequential fourth generation with an extended scalar sector, Phys. Rev. D97 (2018), no. 1 011701, [arXiv: 1707.03000 ].

[24] ATLAS Collaboration, G. Aad et al., Search for pair production of a new heavy quark that decays into a $W$ boson and a light quark in pp collisions at $\sqrt{s}=8 \mathrm{TeV}$ with the ATLAS detector, Phys. Rev. D92 (2015), no. 11 112007, [arXiv:1509.04261].

[25] A. Dighe, D. Ghosh, R. M. Godbole, and A. Prasath, Large mass splittings for fourth generation fermions allowed by LHC Higgs exclusion, Phys. Rev. D85 (2012) 114035, [arXiv: 1204 . 3550 ].

[26] W. Grimus, L. Lavoura, O. M. Ogreid, and P. Osland, A Precision constraint on multi-Higgs-doublet models, J. Phys. G35 (2008) 075001, [arXiv: 0711.4022$].$

[27] W. Grimus, L. Lavoura, O. M. Ogreid, and P. Osland, The Oblique parameters in multi-Higgs-doublet models, Nucl. Phys. B801 (2008) 81-96, [arXiv: 0802 .4353].

[28] G. Bhattacharyya, D. Das, P. B. Pal, and M. N. Rebelo, Scalar sector properties of two-Higgs-doublet models with a global U(1) symmetry, JHEP 10 (2013) 081, [arXiv:1308.4297].

[29] M. Raju, J. P. Saha, D. Das, and A. Kundu, Double Higgs production as an exclusive probe for a sequential fourth generation with wrong-sign Yukawa couplings, arXiv:2001.05280. 